\title{
ESTUDIOS EN 81 CASOS DE BRONCONEUMONIA SARAMPIONOSA
}

\author{
Resultados de un tratamiento standard.
}

\author{
Comunicación preliminar \\ Drs. FERNANDO MARTINEZ, ALBERTO TRAUTMANN \\ y ALFREDO WEIDENSLAUFER \\ Catedra de Pediatría del Prot. A. Atizcia. Hospical "Luis Calvo Mackenna".
}

Han motivado este trabajo dos circunstancias principales: por una parte el aumento de frecuencia de la complicación bronconeumónica en el sarampión, que hemos tenido oportunidad de observar en el Servicio de Infecciosos del Hospital "Luis Calvo Mackenna" a partir de 1952; y por otra, la discordancia de opiniones, tanto en nuestro medio como en la literatura extranjera, en lo que se refiere al criterio terapéutico ${ }^{1-2-3-4-5-6-5-4}$, especialmente la elección del antibiótico. Esto último se vé acrecentado conforme van apareciendo nuevos antibióticos, especialmente los que muestran in vitro un más amplio margen de acción $\mathrm{y}$, por lo tanto, es posible que esa discordancia sea cada vez mayor.

\section{MATERIAL Y MÉTODOS DE ESTUDIO}

Por estas razones y principalmente con el objeto de solucionar en forma práctica este problema en nuestro Servicio, es que decidimos hacer un estudio clínico y terapéutico de esta complicación sobre la base de una pauta más o menos semejante para todos nuestros enfermos en cuanto a observación clínica y tratamiento, a partir de fines de 1952. Logramos, asi, reunir hasta Noviembre de 1954, 81 casos de bronconeumonia sarampionosa, observados y tratados con un mismo criterio $y$ por el mismo personal.

En este material el diagnóstico se fundamentó en los datos proporcionados por la anamnesis y el examen clinico y se complementó con examen radiológico, radioscopías y radiografías cuando se estimó

(WAUke $\times 1$

CASUISTICA POR AFOS Y EDADES

\begin{tabular}{|c|c|c|c|c|c|}
\hline Attoo & $0-1$ & $1-2$ & $2-6$ & mass to 6 & Total \\
\hline $\begin{array}{l}1952 \\
1453 \\
1454\end{array}$ & $\begin{array}{r}2 \\
18 \\
2\end{array}$ & $\begin{array}{r}0 \\
24 \\
5\end{array}$ & $\begin{array}{r}4 \\
68\end{array}$ & $\begin{array}{l}0 \\
2 \\
0\end{array}$ & $\begin{array}{l}6 \\
0,3 \\
13\end{array}$ \\
\hline \multicolumn{2}{|c|}{ TOTAT. $2 \geq$} & $2 y$ & 28 & 2 & 81 \\
\hline
\end{tabular}

necesario, efectuadas al iniciar el tratamiento, al suspenderlo y al alta.

En cuanto a los datos de anamnesis, nos interesaron, fuera de los síntomas, los relacionado con los días de exantema en el momento de aparición de la complicación y también el tratamiento efectuado antes de la hospitalización. Obtuvimos así los datos consignados en los cuadros 2 y 3 .

Estudiadas las bronconeumonias en la forma que expusimos, pudimos separarlas en los siguientes tipos expuestos en el cuadro siguiente, en relación con edad y estado nutritivo

Para decidir la pauta de tratamiento al iniciar este estudio tomamos en consideración varios factores de orden práctico, entre ellos, costo de los antibióticos y el uso de aquellos sin acciones secundarias, tóxicas u otras, y de preferencia de uso paraenteral, a pesar del inconveniente de la inyección, como medio de asegurar su aprovechamiento, especialmente en enfermos graves. No tomamos muy en cuenta el hecho teórico del mayor espectro de acción in vitro y la supuesta y no demostrada acción antivirus, no obstante la probable participación de esta última y de la asociación microbiana en la etiología de la bronconeumonia sarampionosa?.

Por estas razones adoptamos "a priori" el siguiente esquema terapéutico:

1. Al ingreso: penicilina cristalina de 20.000 a 30.000 unidades por Kg. de peso. repartidas cada $6-8$ horas y durante 6 a 7 dias y observación del enfermo por 24 a 48 horas.

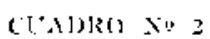

DIAS DE EXANTEMA AL INICIARSE LA BRONCONEUMONIA

\begin{tabular}{ccccc}
\hline $1-3$ & $4-7$ & 8 y más & No nrecisados & Total ' \\
\hline-30 & 54 & 12 & 5 & 81 \\
\hline & & & & \\
\hline
\end{tabular}


(CA)

TRATAMIENTO ANTES DEL INGRESO

\begin{tabular}{|c|c|c|c|}
\hline & Recibieron tsat. & \multirow{2}{*}{$\frac{\text { No recibieron trat. }}{\text { (1+t }}$} & \multirow{2}{*}{$\frac{\text { Total }}{\mathrm{ki}}$} \\
\hline & 17 & & \\
\hline 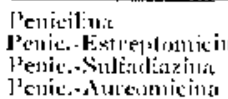 & $\begin{array}{lr}11 \\
\text { inis } \quad \begin{array}{l}3 \\
j \\
1\end{array} \\
j\end{array}$ & & \\
\hline
\end{tabular}

2. Si en este lapso de tiempo no hubiera mejoría desde el punto de vista general $\mathrm{y} / \mathrm{o}$ pulmonar, agregar estreptomicina 50 mgr. por $\mathrm{Kg}$. de peso en 24 horas hasta completar $\operatorname{los} 6$ a 7 días.

3. Si dentro de las 24-48 horas con esta combinación de antibióticos no se obtuviera mejoria, cambiar a terramicina, aureomicina o cloramfenicol $\mathrm{y}$ a iguales dosis aue la estreptomicina.

4. Cuando el enfermo ingresaba con un tratamiento a base de penicilina-estreptomicina por más de 24 horas, se mantenía este tratamiento, ajustando las dosis a las ya enunciadas y aplicando el mismo criterio expuesto.

\section{RESULTADOS}

Para avaluar el resultado consideramos en primer lugar la mejoría general en cuanto a fiebre, estado general, disnea, cianosis, apetito y la regresión de los signos pulmonares físicos y radiológicos. No tomamos en cuenta el tratamiento anterior a Ia hospitalización por el escaso número en que se efectuó, 17, y porque en general fueron dosis insuficientes, 1 a 2 dosis de penicilina y 1 a 2 días de sulfadiazina.

Finalmente, analizamos el número de días de estada en relación con los diversos tratamientos efectuados, tomando en consideración, eso sí, los que debieron permanecer hospitalizados después de curada
Ia complicación pulmonar por otras causas, como otra complicación, por su distrofia o por causas sociales.

Deducimos en primer lugar que de 52 enfermos en que se empezó el tratamiento con penicilina sola $(38$ penicilina, 10 penicilina y después estreptomicina y 4 de los 6 con otros antibióticos), sólo en 10 fué preciso agregar estreptomicina. Al comparar los dos grupos de enfermos no seleccionados cuyo tratamiento se inicio con penicilina 138 ; y con penicilina-estreptomicina $(27)$, ambos semejantes en cuanto al tipo de bronconeumonia, si bien con algunas diferencias favorables para los primeros en cuanto a estado nutritivo, edad y presencia de otras complicaciones, especialmente laringotraqueítis (cuadro $N^{\circ} 8$, puede verse que no hubo variaciones marcadas en cuanto al plazo en que se produjo la mejoría clínica y radiolńgica $\mathrm{y}$ en cuanto a días de hospitalización.

En los 10 casos en que fué preciso agregar estreptomicina y que por lo tanto fueron más graves que los primeros con penicilina exclusiva, la mejoría clínica $y$ radiológica fué más tardía, como también más prolongada la hospitalización. Entre ellos fueron también más frecuentes los tipos de bronconeumonia difusas y más numerosos los niños con mal estado nutritivo.

Finalmente, no creemos poder sacar deducciones del grupo de 6 niños tratados con terramicina (5) y cloramfenicol (1), sino solamente exponer nuestra impresión en el sentido de que siendo 5 de ellos graves, especialmente por tratarse de la forma bilateral difusa, la mayoría de los niños menores ( 4 lactantes y 2 pre-escolares i con mal estado nutritivo (4) y con otras complicaciones 15 ), la mejoria clínica y radiológica de la complicación que nos ocupa fué semejante a la del grupo

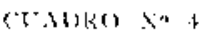

FORMAS DE BRONCONEUMONIA, EDAD Y ESTADO NUTRITIVO

\begin{tabular}{|c|c|c|c|c|c|c|c|c|c|c|c|}
\hline Años & 1 & & $3 \mathrm{fi}_{\mathrm{i}}$ & & $! !$ & & 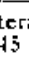 & & & 1 & $\begin{array}{c}\text { Total } \\
\therefore 1\end{array}$ \\
\hline Edad & \rfloor & Difusas & I & Circungtritas & $!$ & Difusas & | & & Circunscrilas & I & \\
\hline $\begin{array}{r}0 \\
1 \\
2\end{array}-\frac{1}{2}$ & 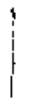 & $\begin{array}{l}3 \\
2 \\
5 \\
0\end{array}$ & i & $\begin{array}{r}1: \\
i \\
7 \\
1\end{array}$ & $\begin{array}{l}1 \\
1 \\
1 \\
1 \\
1 \\
1\end{array}$ & $\begin{array}{r}8 \\
19 \\
1, i \\
I\end{array}$ & 1 & & $\begin{array}{l}\text { 1) } \\
1 \\
3 \\
1 \\
1\end{array}$ & 1 & \\
\hline Motal. & I & 10 & $i$ & 20 & 11 & 41 & $!$ & & if & ' & 81 \\
\hline $\begin{array}{l}\text { Esitaclo } \\
\text { mutritifu: } \\
\text { Jiacus } \\
\text { Dlato }\end{array}$ & i & $\frac{5}{5}$ & 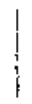 & $\begin{array}{l}11 \\
15\end{array}$ & $\begin{array}{l}1 ! \\
11\end{array}$ & 3 & 1 & - & $\begin{array}{l}0 \\
4\end{array}$ & 1 & $\begin{array}{l}2.3 \\
.36\end{array}$ \\
\hline
\end{tabular}


(1) (N) No No 5

TRATAMIENTO Y FORMAS DE BRONCONEUMONIA

\begin{tabular}{|c|c|c|c|c|c|c|c|c|c|c|}
\hline & 1 & & 11 & \multicolumn{3}{|c|}{ Unilatcral } & 1 & \multicolumn{3}{|c|}{ B i l a t e $r$ a 1} \\
\hline & & Total & $\therefore$ & Difusa & I & Circunscrita & I & Difusa & I & Circunscrita \\
\hline l'culciljua & I & $3 k$ & $\because$ & 5 & 1 & 14 & I & 13 & 1 & 1 \\
\hline J'ensidilind- Estrajutgnticinat & I & 27 & II & $\vec{s}$ & I & $\theta$ & ! & 12 & 1 & 1 \\
\hline l'entic, y destmés Estrejtuntic. & & 11 & 1 & $\mathrm{u}$ & I & 2 & $!$ & 6 & 1 & 2 \\
\hline Gotros antilointicus & I & 6 & II & ( ) & i & 1 & I & j & 1 & 0 \\
\hline T'OC'Al & 1 & 81 & $\cos u s$ & & & & & & & \\
\hline
\end{tabular}

CLIJRO N" 6

RESULTADO DEL TRATAMIENTO CON LOS DISTINTOS ANTIBIOTICOS, TIPO DE BRONCONEUMONIA. ESTADO NUTRITIVO, TDAD Y DIAS DE HOSPITALIZACION

\begin{tabular}{|c|c|c|c|c|c|c|c|c|c|c|c|c|c|c|c|c|c|c|c|}
\hline \multirow{3}{*}{ Antibiótico } & \multicolumn{2}{|c|}{$\begin{array}{l}\text { N!de } \\
\text { casos }\end{array}$} & \multicolumn{6}{|c|}{ Bronconeumonia } & \multirow{2}{*}{\multicolumn{3}{|c|}{$\mid \begin{array}{c}\text { Ertado } \\
\text { nutritivo }\end{array}$}} & \multirow{2}{*}{\multicolumn{3}{|c|}{ Edad }} & \multirow[b]{2}{*}{ en } & \multirow[b]{2}{*}{ ax̃os } & \multirow[b]{2}{*}{ Alt: } & \multirow[b]{2}{*}{ th } & \multirow[b]{2}{*}{ días } \\
\hline & & & \multicolumn{3}{|c|}{ Bilateral } & & \multicolumn{2}{|c|}{ Unilatc:al } & & & & & & & & & & & \\
\hline & & & $i$ & Circ. & Dif. & । & Circ. & Dif. & I & B. & M. & 10 & & $1-2$ & $2-6$ & $\dashv$ de 6 ! & 1.10 & $11-20$ & + de 20 \\
\hline L'enicilinat & I & .3 .5 & ' & 1 & $1 s$ & $:$ & 14 & $s$ & 1 & 14 & 24 & 11 & & () & 17 & 2 & $1 . i^{*}$ & $1 r_{1}$. & 8 \\
\hline Peuic, y Estrept. & $!$ & 27 & I & 1 & 12 & I & 9 & $i$ & I & 7 & $2+k$ & I & 7 & $\mathrm{t} \vec{s}$ & $\overrightarrow{\mathfrak{i}}$ & 1] & $7^{*}$ & I6 & 3 \\
\hline l'eúc, $y$ tesipués fistrept. & 1 & 10 & । & 3 & i & I & 2 & 9 & $\cdot$ & 2 & 8 & $1+$ & -1 & 2 & + & 0 & $!$ & 5 & 5 \\
\hline ()tros alltilioticose & I & 6 & $\vdots$ & u & 5 & 1 & 1 & $n$ & i & 2 & $t$ & 1 & 1 & 3 & 2 & 0 & 1 & 1 & 3 \\
\hline TOTAI & i & 81 & $:$ & 4 & 41 & 1 & 26 & li! & 1 & $2 \vec{j}$ & $56 \mathrm{i}$ & 12 & & $2 \%$ & 28 & 2 & 22 & $3 \mathrm{~s}$ & $j y$ \\
\hline & 1 & & & & & $\$ 1$ & & & 1 & & & i & & si & & & i & 81 & \\
\hline
\end{tabular}

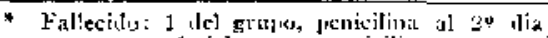

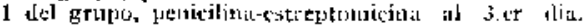

(CADRO NH:

MEJORIA CLINICA Y RADIOLOGICA DE LA BRONCONEUMONIA

\begin{tabular}{|c|c|c|c|c|c|c|c|c|c|c|c|c|c|c|c|c|}
\hline \multirow{3}{*}{ Antibiótico } & & \multirow{3}{*}{$\begin{array}{l}\text { Nथ de } \\
\text { casos }\end{array}$} & \multicolumn{4}{|c|}{ Bronconeuraonia } & \multirow{2}{*}{ 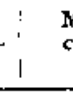 } & \multirow{2}{*}{$\begin{array}{l}\text { Mejoría } \\
\text { cinica. } \\
\text { Dias }\end{array}$} & \multirow{3}{*}{ i } & \multirow{2}{*}{\multicolumn{3}{|c|}{$\begin{array}{c}\text { Mejoria } \\
\text { radiơógica. } \\
\text { Días }\end{array}$}} & \multirow{2}{*}{\multicolumn{2}{|c|}{$\begin{array}{c}\text { No } \\
\text { conteo } \\
\text { lado }\end{array}$}} & 1 & \multirow{3}{*}{$\begin{array}{l}\text { Falle- } \\
\text { cidos }\end{array}$} \\
\hline & & & \multicolumn{2}{|c|}{ Unilateral } & \multicolumn{2}{|c|}{ Bilateral } & & & & & & & & & & \\
\hline & I & & 1 Circ. & Dif. & Citc. & Dif. & 0.7 & $+\mathrm{de} ?$ & & & & & I & & 1 & \\
\hline f'enicitinas & $i$ & 38 & 14 & 5 & $\mathbf{1}$ & is & 128 & 9 & I & 7 & 15 & 1 & I & 15 & 1 & 1 \\
\hline I'elric. $y$ Estrert. & 1 & 27 & 9 & $\overrightarrow{5}$ & 1 & 12 & 13 & 17 & 1 & 5 & 13 & 3 & I & 7 & 1 & $\mathbf{I}$ \\
\hline l'enic, $y$ tlesplućs Estrejt. & . & 11 & 2 & 3 & 2 & $t$ & 2 & 3 & ! & 11 & 3 & $\overrightarrow{\mathbf{s}}$ & I & 2 & I & 0 \\
\hline Otros antibiúlicos & I & 0 & 1 & 0 & 0 & 5 & $! 4$ & 2 & I & 2 & 2 & 1 & 1 & 1. & 1 & it \\
\hline \multirow[t]{2}{*}{ TUTAL } & ; & 81 & 26 & 19 & 1 & 41 & 43 & 36 & I & $1+$ & 33 & $s$ & I & 25 & 1 & 2 \\
\hline & 1 & & i & & \$1 & & & $7 y$ & I & & & $3 y$ & & & & \\
\hline
\end{tabular}

$([-A) R O)$ No"s

BRONCONEUMONIA SARAMPIONOSA. OTRAS COMPLICACIONES

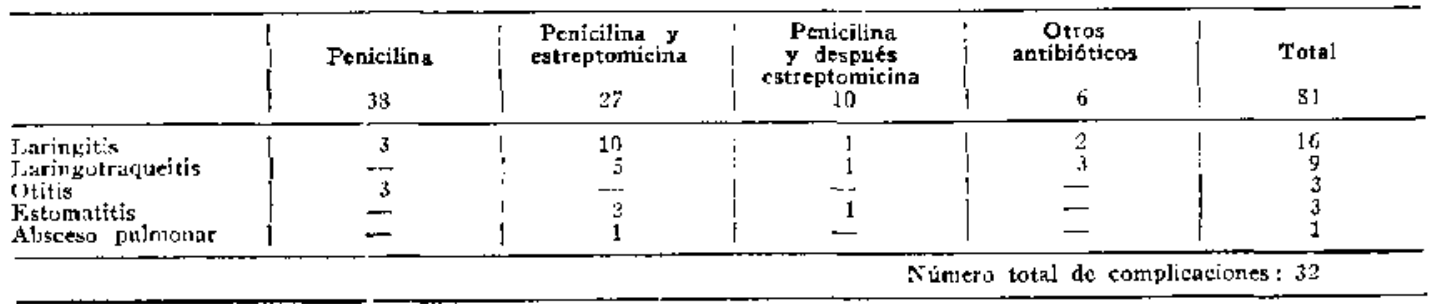


con penicilina. En este grupo con otros antibióticos tratamos 2 de las 3 recaídas de bronconeumonia de nuestra casuística, cuyo resumen damos a continuación:

Pedro C. Obs. 53-19464. Lactante distrófico de un año 2 meses, pess $7.850 \mathrm{gt}$., ingresa el 21 -VIIL-53 al 3.er día de exantema sarampionoso, y con bronconeumonia. crépitos $\mathrm{g}$ subcrépitos en las bases. tratado desde el dia anterior con $400.000 \mathrm{U}$. de penicilina tapi-lenta. En nuestro Servicio recibe penicilina 7 dias. Al $8^{\prime \prime}$ dia aparece condensación del vértice derecho. que esta vez se trata con terramicina, con mejoría clinica a los 5 dias. Es dado de alta a los 17 dias. persistiendo imagen tenne de condensación a radioscopia. Diagnóstico: Sarampión. Bronconqumonia.

Luis V. Obs. 52-8123. Lactante distrófico de 1 año $91 / 2$ meses, con $9.180 \mathrm{gr}$. Enferma de sarampión el 28.VIII-53 y el 3-IX se diagnostica bronconeumonia sarampionosa derecha y laringitis. Reclbe 60.000 U. de penicilina cada 6 horas 1 día $y$ es hospitali. zado al día siguiente, siendo tratado ton penicilinaestreptomicina 7 dias. facha de la mejoría slínica. Reingresa el 22-IX.53 por bronconeumonia derecha, 2/3 superiotes. Esta vez se le trasa con penicilina 5 dias. observándose mejoria elinica $y$ radiológica al $7^{\circ}$ dia (cisuritis) .

Sergio S. Obs. 52-7902. Lactante distrófico de 1 año 5 meses, con 7.520 grs., presenta sarompión el $12 \cdot V \cdot 53,2$ días después consulta a Policlínica. donde se comprueba bronconeumonia bilateral, trarándosele con penicilina rapi-lentz $\mathbf{4 0 0 . 0 0 0} \mathrm{U}$. diarias, acusando

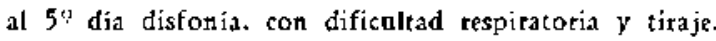
Con los diagnósticos de convaleciente de sarampión, đistrofia, bronconeumonia y laringitis, se hospitaliza y se trata con $100.000 \mathrm{U}$. de penicilina cada 6 horas. La laringoscopia revela difteria faringea por lo que recibe $20.000 \mathrm{U}$. de antitoxina difterica. Al $2^{\circ}$ dia, por continuar grave, se cambia $J a$ penicilina por terramicina, que recibe 7 días. A los 10 días está afebril. la mejoría clínica de la bronconeumonia se presenta a los 12 días y es dado de alta a los 21 días por habet presentado descamación de tipo escarlatinoso $y$ haberse comprobada estreptococo hemolítico en la se. creción faringea. Reingresa 10 dias después ron bronconeumonia bilateral, ctépitos y subcrépitos en ambos campos pulmonares, respiración soplante del $1 / 3$ superior izquietdo. Recibe peatcilina $60.000 \mathrm{U}$. cada 6 horas. At $6^{\circ}$ dia. por haberse observado a rayos $\mathrm{X}$ imágenes negativas interpretadas coma abscesos pulmonares (1uberculinas (-), se agrega estreptomicina $200 \mathrm{mgr}$. cada 12 horas por 9 días. Cae la fiebre al 3.er día de este tratamiento y mejora lentamente, encontrándose normalidad a rayos $X$ en controles posteriores. siendo dado de alta a los 28 días con los siguientes diagnósticos: distrofia, otitis supurada, bronconeumonia y abscesos pulmonares.
A continuación resumimos las observaciones de los 2 fallecidos:

Jaime S. Obs. 53-22101. Se trata de un lactance que consulta a Policlínica al $4^{\circ}$ día del exantema sa. rampionoso por disnea quejumbrosa y disfonía, recibiendo $200.000 \mathrm{U}$. de penicilina cada 12 horas y estreptomitina $1 \mathrm{gr}$. en 24 horas. hospitalizado al dia siguiente por intensificación de su cuadra laríngeo, siendo meccsario practicar traquzotomia y suministrar 02. perícilina $200.000 \mathrm{U}$. cada 8 horas y estreptomicina $150 \mathrm{mgr}$ cada 8 horas. Fallece al 3.er dia. La anatomia patolúgica revela sarampión, laringo-tráqueobronquitis aguda, bronconeumonia alveolar $e$ intersticial e infiltración grasosa del higado.

Cacilia A. Obs. 52-9985. Lactante de 1 año 2 meses. distrófica, ingresa el 29-I-53, enfertha 2 dias con sarampión. se trata I dia con penicilina $200.000 \mathrm{U}$. c ingresa inconsciente, con fiebre de $42^{\circ}$, deshidratada, con empastamiento $y$ convulsiones tónico-clónicas generalizadas. Con diagnósticos de distrofia. encefalitis sarampionosa y convulsiones por hipettermia se le hospicaliza, tratándose con aspirina $0.10 \mathrm{gr}$. luminal 0.03 gr. y penicilina 100.000 U. cada 6 horas. Continia muy geave. con conculsiones e hipertonía y fallece al $2^{\circ}$ día. La autopsia resela congestión de todos los ótganos, encéfalo con intensa hiperemia, cianosis y pequeñas hemortagias, focos de bronconeumonia. Díagnóstito: sarampión, bronconeumonia, encefalitis.

Desde el punto de vista general pudimos comprobar la mayor frecuencia de los tipos bilaterales difusos de bronconeumonia (41), la frecuencia con que se ásocia a otras complicaciones (32) y dentro de éstas la laringotraqueítis. También la mayor frecuencia en la época de lactantes (51), antes de los 2 años, y el gran predominio en los desnutridos $(56)$, si bien asi lo es en la mayoría de los niños que consultan en nuestro hospital. Su mayor incidencia (64) se produce en la primera semana que sigue a la aparición del exantema.

\section{RESUMEN $Y$ CONCLUSIONES}

1. Se analizan 81 casos de bronconeumonia sarampionosa ingresados entre 1952 y 1954 inclusive en el Servicio de Infecciosos del Hospital "Luis Calvo Mackenna". Se estudian clínicamente con el mismo criterio y tratan siguiendo una pauta uniforme.

2. Esta complicación aparece como más frecuente en los lactantes y niños desnutridos en las distintas épocas de la vida, 
siendo el periodo de mayor incidencia los siete dias siguientes a la aparición del exantema, ligeramente mayor en los últimos tres días.

3. En la mitad de los casos (41) se observó un tipo de bronconeumonia grave bilateral y difusa y en más de la tercera parte $\{32$ ) asociada a otras complicaciones, sobre todo a laringotraqueitis (25).

4. A pesar del rol etiológico posible del virus del sarampión y/o de la asociación microbiana de gérmenes en la etiología de esta complicación, la penicilina a dosis corrientes, permitió, en nuestro material, obtener resultados que consideramos eficaces.

5. En los casos controlados, la mejoria clínica de la bronconeumonia precedió a la mejoría radiológica.

6 . En el pronóstico de nuestro material desempeñaron un papel más importante aue el tipo de bronconeumonia la edad, estacio nutritivo y presencia de otras complicaciones o enfermedades concomitantes.

\section{SUMMARY AND CONCLUSIONS}

1. Eighty-one cases of measles bronchopneumonia are reported, all of them admitted in the Contagious Department of the Luis Calvo Mackenna Children's
Hcspital between 1952 and 1954, treated and analized on the same bases.

2. This complication occurs with a high incidence in malnourished infants and children at different ages and more frecuently in the first three to seven days after the beginning of the exenthem.

3. A severe bilateral bronchopneumonia was observed in one half of the cases (41). Other complications, especially laryngotracheitis, were seen in more than one third of them.

4. Regular doses of penicillin were enough to obtain satisfactory results in spite of the possibility of virus and/or bacterial association in the etiology of this complication.

5. Age, nutritional condition and incidence of other complications were more important in the prognosis than the type of bronchopneumonia.

\section{BIBLIOGRAFİA}

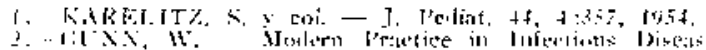
2. 1:0.

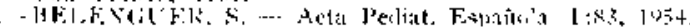

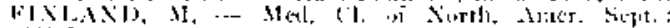
$12,59-32,1952$

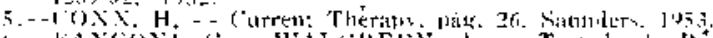

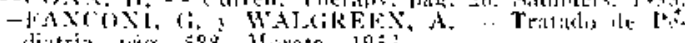

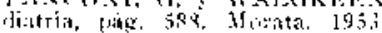

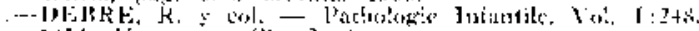
195: X[assun et Cit. ]'aris

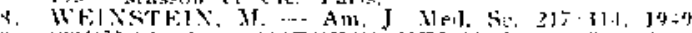

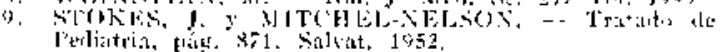

